\title{
Hard scale uncertainty in collinear factorization: Perspective from $\boldsymbol{k}_{t}$-factorization
}

\author{
Benjamin Guiot* \\ Departamento de Física, Universidad Técnica Federico Santa María, Casilla 110-V, Valparaiso, Chile
}

(Received 24 April 2018; published 25 July 2018)

\begin{abstract}
We analyze two consequences of the relationship between collinear factorization and $k_{t}$-factorization. First, we show that the $k_{t}$-factorization gives a fundamental justification for the choice of the hard scale $Q^{2}$ done in the collinear factorization. Second, we show that in the collinear factorization there is an uncertainty on this choice which will not be reduced by higher orders. This uncertainty is absent within the $k_{t}$-factorization formalism.
\end{abstract}

DOI: 10.1103/PhysRevD.98.014036

\section{INTRODUCTION}

At very high energies, the $k_{t}$-factorization $[1,2]$ or semihard approach $[3,4]$ is believed to be the correct formulation. It is more general than the collinear factorization, and it is well known that the latter is obtained in the $k_{t}^{2} \rightarrow 0$ limit of the former $[1,2,4]$. If the unintegrated gluon density obeys the Balitsky-Fadin-Kuraev-Lipatov (BFKL) equation [5], it resums terms proportional to $\alpha_{s} \ln \left(\frac{1}{x}\right)$, retaining the full $Q^{2}$ dependence and not just the leading $\ln Q^{2}$ terms [6,7].

One advantage of the $k_{t}$-factorization is a better treatment of the kinematics, using unintegrated parton densities and off-shell matrix elements (meaning that the parton virtualities, which can be easily larger than $100 \mathrm{GeV}^{2}$ at the LHC, are not neglected). Moreover, the use of unintegrated parton densities implies that, even at leading order, outgoing partons are not back to back in the laboratory frame. To obtain this qualitative result with the collinear factorization, it is necessary to go to higher orders (but their computation is easier than in the $k_{t}$-factorization). In Ref. [7], one can find a table comparing collinear and $k_{t}$-factorization for different non-inclusive observables.

Here we want to discuss another advantage of the $k_{t}$-factorization, namely, the disappearance of an uncertainty present in the collinear factorization. In the latter, the cross section depends on the center-of-mass energy $\sqrt{s}$, the hard scale $Q^{2}$, and the factorization scale $\mu$ (and on the renormalization scale, which will be ignored in this study). In hadron-hadron collisions, the conventional choice for

\footnotetext{
*benjamin.guiot@usm.cl
}

Published by the American Physical Society under the terms of the Creative Commons Attribution 4.0 International license. Further distribution of this work must maintain attribution to the author(s) and the published article's title, journal citation, and DOI. Funded by SCOAP ${ }^{3}$. differential cross sections is $Q^{2} \sim p_{t}^{2}$ with $p_{t}$ the transverse momentum of the outgoing partons in the center-of-mass frame. While current calculations take into account the well-known factorization scale uncertainty, nothing is said about the choice of the hard scale.

The two main results are given in Secs. IV and V. In Sec. IV, using the $k_{t}$-factorization, we demonstrate that there is an uncertainty in the collinear factorization, coming from the choice of the hard scale. This choice being not necessary in the case of $k_{t}$-factorization, the discussed uncertainty is absent in this formalism. Contrary to the factorization scale, the hard scale uncertainty is not reduced by higher-order calculations. In Sec. V, we show that the choice $Q^{2} \sim p_{t}^{2}$ can be justified by the dynamical behavior of the off-shell cross sections and unintegrated parton densities used in the $k_{t}$-factorization.

\section{COLLINEAR FACTORIZATION AND UNCERTAINTIES}

For hadron-hadron collisions, the collinear factorization formula is generally written. ${ }^{1}$

$$
\begin{aligned}
& \frac{d \sigma}{d x_{1} d x_{2} d p_{t}^{2}}\left(x_{1}, x_{2}, p_{t}^{2}, Q^{2}, \mu^{2}\right) \\
& \quad=f\left(x_{1}, \mu^{2}\right) f\left(x_{2}, \mu^{2}\right) \hat{\sigma}\left(x_{1}, x_{2}, p_{t}^{2}, \frac{Q^{2}}{\mu^{2}}\right) .
\end{aligned}
$$

The functions $f$ and $\hat{\sigma}$ are the parton densities and partonic cross sections, respectively. The variable $p_{t}$ corresponds to the transverse momentum of outgoing partons in the center-of-mass frame. We use the generic notation $Q^{2}$ for the hard scale which is conventionally identified with $p_{t}^{2}$.

\footnotetext{
${ }^{1}$ We will use mainly schematic formulas. The sum over parton flavors is ignored, and one can consider that there is only one flavor [it simplifies also the Dokshitzer-Gribov-Lipatov-AltarelliParisi (DGLAP) equation]. If not necessary, integrals are not written.
} 
The factorization scale $\mu$ appears due to the renormalization procedure. It comes inside logarithms of the type $\alpha_{s} \ln \left(Q^{2} / \mu^{2}\right)$ and has to be chosen close to $Q^{2}$ for an accurate finite order calculation. The dependence on the renormalization scale is not shown, and in this study we take $\alpha_{s}$ constant. Finally, the longitudinal momentum fractions $x_{i}$ carried by the incoming partons are given by

$$
x_{1}=\frac{p_{a, t}}{\sqrt{s}} e^{y_{a}}+\frac{p_{b, t}}{\sqrt{s}} e^{y_{b}}, \quad x_{2}=\frac{p_{a, t}}{\sqrt{s}} e^{-y_{a}}+\frac{p_{b, t}}{\sqrt{s}} e^{-y_{b}},
$$

with $a$ and $b$ referring to the two outgoing partons, $y_{i}$ the rapidities, and $s$ the Mandelstam variable for the hadronic system.

The definition of parton densities is not unique [8], and, for our discussion, it is simpler to shift higher-order corrections from $\hat{\sigma}$ to these functions, leading to the following factorization formula:

$$
\begin{aligned}
& \frac{d \sigma}{d x_{1} d x_{2} d p_{t}^{2}}\left(x_{1}, x_{2}, p_{t}^{2}, Q^{2}, \mu^{2}\right) \\
& \quad=f\left(x_{1}, Q^{2} ; \mu^{2}\right) f\left(x_{2}, Q^{2} ; \mu^{2}\right) \hat{\sigma}\left(x_{1}, x_{2}, p_{t}^{2}\right) .
\end{aligned}
$$

Taking into account the first higher-order corrections and following Ref. [6], we write

$$
\begin{aligned}
f\left(x, Q^{2} ; \mu^{2}\right)= & f\left(x, \mu^{2}\right)+\frac{\alpha_{s}}{2 \pi} \int_{x}^{1} \frac{d \xi}{\xi} f\left(\xi, \mu^{2}\right) \\
& \times\left(P\left(\frac{x}{\xi}\right) \ln \frac{Q^{2}}{\mu^{2}}+C(x)\right),
\end{aligned}
$$

with $C(x)$ a calculable function which is not enhanced by $\ln \left(Q^{2} / \mu^{2}\right)$. In the following, we will keep the choice and notation of Eqs. (3) and (4).

In an all-order calculation, the dependence on the unphysical scale $\mu$ will disappear in both sides of Eq. (3). This is formalized by the DGLAP equation [9] (or Ref. [6] for a modern review), which can be written

$$
\frac{d f\left(x, Q^{2} ; \mu^{2}\right)}{d \mu^{2}}=0 .
$$

However, in perturbation theory this equation is exact only at a given order, and the parton densities have, in fact, a dependence on $\mu$ due to higher-order corrections, justifying our notation. In the opposite, in the r.h.s. of Eq. (4), $f\left(x, \mu^{2}\right)$ is given to all orders (by a measurement) and depends only on two variables. It is interesting to look at the solution of the DGLAP equation in the $N$-moment space. Inserting (4) in (5) and taking the Mellin transform, we obtain

$$
q^{2} \frac{\partial f_{N}\left(q^{2}\right)}{\partial q^{2}}=\frac{\alpha_{s}}{2 \pi} \gamma_{N} f_{N}\left(q^{2}\right)+\mathcal{O}\left(\alpha_{s}^{2}\right)
$$

where $\gamma_{N}$ is the anomalous dimension and, for simplicity, we consider $\alpha_{s}$ constant. The solution is then given by

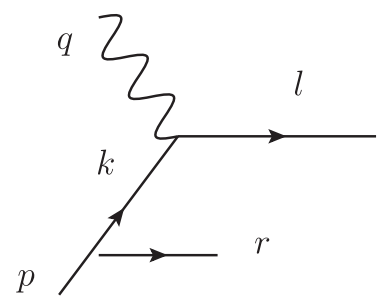

FIG. 1. Real emission diagram in DIS.

$$
\begin{aligned}
f_{N}\left(Q^{2} ; \mu^{2}\right) & =\exp \left[\int_{\mu^{2}}^{Q^{2}} \frac{\partial q^{2}}{q^{2}} \frac{\alpha_{s}}{2 \pi} \gamma_{N}\right] f_{N}\left(\mu^{2}\right) \\
& =\left(\frac{Q^{2}}{\mu^{2}}\right)^{\frac{\alpha_{s}}{2 \pi} \gamma_{N}} f_{N}\left(\mu^{2}\right)
\end{aligned}
$$

where we can see the dependence of the parton densities on $Q^{2}$ and $\mu^{2}$.

One remark on the hard scale $Q^{2}$ before the discussion on uncertainties is in order. The logarithm $\ln \left(Q^{2} / \mu^{2}\right)$ arises from an integral on the transverse momentum, $k_{t}$, of the incoming parton [an example is given in the case of deep inelastic scattering (DIS) in Fig. 1]. The hard scale appears in the upper bound of such an integral.

In this paper, we want to discuss the usual choice ${ }^{2}$ $Q^{2}=p_{t}^{2}$ done in hadron-hadron collisions, giving the factorization formula

$$
\begin{aligned}
& \frac{d \sigma}{d x_{1} d x_{2} d p_{t}^{2}}\left(x_{1}, x_{2}, p_{t}^{2}, \mu^{2}\right) \\
& \quad=f\left(x_{1}, p_{t}^{2} ; \mu^{2}\right) f\left(x_{2}, p_{t}^{2} ; \mu^{2}\right) \hat{\sigma}\left(x_{1}, x_{2}, p_{t}^{2}\right) .
\end{aligned}
$$

For definiteness, we consider the case of transverse momentum distribution of heavy quarks in proton-proton collisions at the LHC. This choice for the hard scale means that $p_{t}^{2}$ is assumed to be the upper bound for the $k_{t}^{2}$ integration. Since for on-shell partons the kinematical constraint is $k_{t}^{2}<\hat{s} / 4$ (with $\hat{s}=x_{1} x_{2} s$ ), this is a good approximation in the region $p_{t}^{2} \simeq \hat{s} / 4$, but it is not correct if $\Lambda_{\mathrm{QCD}}^{2} \ll p_{t}^{2} \ll \hat{s} / 4$. In fact, it is exactly in this region that the $k_{t}$-factorization is expected to give important corrections.

In the following, we will argue that the $k_{t}$-factorization provides a fundamental explanation on why choosing the hard scale to be $p_{t}^{2}$ is correct. But we will also see that this choice is not unique and gives rise to a theoretical uncertainty (in the collinear factorization case) which is not taken into account in current calculations. This uncertainty is not reduced by higher-order corrections. The other uncertainties come from the choice of the factorization scale, the mass, and parton densities.

\footnotetext{
${ }^{2} \operatorname{Or} Q^{2}=m_{t}^{2}$, with $m_{t}^{2}=p_{t}^{2}+m^{2}$ and $m$ the mass of the outgoing parton(s).
} 


\section{III. $\boldsymbol{k}_{\boldsymbol{t}}$-FACTORIZATION}

The $k_{t}$-factorization (sometimes called high-energy factorization or the semihard approach) has been developed in parallel in Refs. [1-4]. In order to include all theoretical and phenomenological studies, we define the $k_{t}$-factorization as a convolution of unintegrated gluon densities with offshell cross sections. For hadron-hadron collisions, it can be written

$$
\begin{aligned}
& \frac{d \sigma}{d x_{1} d x_{1} d^{2} p_{t}}\left(s, x_{1}, x_{2}, p_{t}^{2}, \mu^{2}\right) \\
& =\int^{k_{\max }^{2}} d^{2} k_{1 t} d^{2} k_{2 t} F\left(x_{1}, k_{1 t}^{2} ; \mu^{2}\right) F\left(x_{2}, k_{2 t}^{2} ; \mu^{2}\right) \\
& \quad \times \hat{\sigma}\left(x_{1} x_{2} s, k_{1 t}^{2}, k_{2 t}^{2}, p_{t}^{2}\right) .
\end{aligned}
$$

The variables $k_{1 t}, k_{2 t}, x_{1}$, and $x_{2}$ refer to the two spacelike partons entering in the $2 \rightarrow 2$ perturbative QCD process. They correspond to the transverse momentum and the hadron longitudinal momentum fraction. The variable $p_{t}$ is for the transverse momentum of the outgoing parton. The precise definition for the upper bound $k_{\max }^{2}$ will be given in another publication; here it is sufficient to know that

$$
k_{\max }^{2}>x_{1} x_{2} s / 4=p_{t, \max }^{2} .
$$

In this paper, the only restriction on the unintegrated gluon density $F\left(x, k_{t}^{2} ; \mu^{2}\right)$ is that the second scale $\mu^{2}$ has to be interpreted as the factorization scale. In this case, it is related to the usual gluon density by

$$
f\left(x, Q^{2} ; \mu^{2}\right)=\int^{Q^{2}} F\left(x, k_{t}^{2} ; \mu^{2}\right) d^{2} k_{t},
$$

where we follow the notation used in Refs. [10,11]. ${ }^{3}$ For completeness, we mention that, for practical purposes, a specific treatment has to be done in the infrared. Some examples can be found in Refs. [12,13]. The unintegrated gluon density can be obtained by inverting relation (11):

$$
F\left(x, k_{t}^{2} ; \mu^{2}\right)=\frac{1}{\pi} \frac{\partial f\left(x, k_{t}^{2} ; \mu^{2}\right)}{\partial k_{t}^{2}} .
$$

The corresponding equation in the $N$-moment space is

$$
F_{N}\left(k_{t}^{2} ; \mu^{2}\right)=\frac{\alpha_{s}}{2 \pi^{2} k_{t}^{2}} \gamma_{N}\left(\frac{k_{t}^{2}}{\mu^{2}}\right)^{\frac{\alpha_{s}}{2 \pi} \gamma_{N}} f_{N}\left(\mu^{2}\right),
$$

where Eq. (7) has been used. Equation (9) is, for instance, valid for Kimber-Martin-Ryskin [14] and BFKL unintegrated gluon densities. In the latter case, an expression for $F_{N}\left(k_{t}^{2} ; \mu^{2}\right)$ can be found in Refs. [10,11]. The factor $\frac{\alpha_{s}}{2 \pi} \gamma_{N}$ is replaced by $\gamma_{N}\left(\alpha_{s}\right)$, which has a perturbative expansion in $\alpha_{s} / N$, first obtained by Balitsky, Fadin, Kuraev, and Lipatov [5].

\footnotetext{
${ }^{3}$ However, our function $F\left(x, k_{t}^{2} ; \mu^{2}\right)$ is related to their function by a factor of $x$.
}

The cross section $\hat{\sigma}$ is computed using off-shell matrix elements (see [7] for more details). We will discuss the case where outgoing partons are on shell and the two incoming partons are spacelike, with off-shellness $k_{1}^{2} \simeq-k_{1 t}^{2}$ and $k_{2}^{2} \simeq-k_{2 t}^{2}$. We will see that taking into account this degree of freedom (which requires additional integrations on $\vec{k}_{t}$ ) is the reason behind the disappearance of the uncertainty on $Q^{2}$, presented in the previous section.

We will close this section by two remarks. The most general expression for $F_{N}\left(k_{t}^{2} ; \mu^{2}\right)$ derived in Ref. [15] in the $N \rightarrow 0$ limit and minimal subtraction scheme is

$$
F_{N}\left(k_{t}^{2} ; \mu^{2}\right)=R\left(\alpha_{s}\right) \frac{\gamma_{N}\left(\alpha_{s}\right)}{\pi}\left(\frac{k_{t}^{2}}{\mu^{2}}\right)^{\gamma_{N}\left(\alpha_{s}\right)} f_{N}^{\overline{\mathrm{MS}}}\left(\mu^{2}\right),
$$

with $R\left(\alpha_{s}\right)$ having the following perturbative expansion:

$$
\begin{aligned}
R\left(\alpha_{s}\right)= & 1+\frac{8}{3} \zeta(3)\left(\frac{\bar{\alpha}_{s}}{N}\right)^{3}-\frac{3}{4} \zeta(4)\left(\frac{\bar{\alpha}_{s}}{N}\right)^{4} \\
& +\frac{22}{5} \zeta(5)\left(\frac{\bar{\alpha}_{s}}{N}\right)^{5}+\mathcal{O}\left(\left(\frac{\bar{\alpha}_{s}}{N}\right)^{6}\right)
\end{aligned}
$$

with $\bar{\alpha}_{s}=C_{A} \alpha_{s} / \pi$ and $\zeta(n)$ the Riemann zeta function. The expression given for $F_{N}\left(k_{t}^{2} ; \mu^{2}\right)$ in Eq. (13) corresponds to the lowest order $(R=1)$.

The second remark is that one can encounter the following definition:

$$
f\left(x, \mu^{2}\right)=\int^{\mu^{2}} d^{2} k_{t} F\left(x, k_{t}^{2}, \mu^{2}\right) .
$$

By writing the 1.h.s. $f\left(x, \mu^{2} ; \mu^{2}\right)$, we can see that this is nothing else than our definition (11) with the choice $Q^{2}=\mu^{2}$.

\section{RELATIONSHIP BETWEEN COLLINEAR AND $\boldsymbol{k}_{\boldsymbol{t}}$-FACTORIZATION: DISCUSSION ON THE HARD SCALE UNCERTAINTY}

In Sec. II, we discussed the fact that in the collinear factorization a choice for $Q^{2}$ has to be done and that it should be accompanied by an uncertainty. The reason why this uncertainty is absent in the $k_{t}$-factorization is because the transverse momentum dependence of the incoming partons is explicitly taken into account and integrated up to the kinematical upper bound $k_{\max }^{2}$; cf. Eq. (9). It is not necessary to choose the physical scale inside the unintegrated parton densities, since all possibilities are taken into account, "weighted" by the $k_{t}$-dependent off-shell cross section.

To understand why, in Eq. (8), the scale inside the parton density is approximatively $p_{t}^{2}$ and why the collinear factorization still works ${ }^{4}$ at $p_{t}^{2} \ll s$, it is interesting to see how the collinear factorization can be found as a limit of the $k_{t}$-factorization. Equation (9) can be written

\footnotetext{
${ }^{4}$ To be precise on this statement, one should specify the process under consideration. Here we mean that, for sufficiently inclusive quantities, there is no huge discrepancy.
} 


$$
\begin{aligned}
\frac{d \sigma}{d x_{1} d x_{2} d p_{t}^{2}}= & \int^{p_{t}^{2}} d^{2} k_{1 t} d^{2} k_{2 t} F\left(x_{1}, k_{1 t}^{2} ; \mu^{2}\right) \\
& \times F\left(x_{2}, k_{2 t}^{2} ; \mu^{2}\right) \hat{\sigma}\left(x_{1} x_{2} s, k_{1 t}^{2}, k_{2 t}^{2}, p_{t}^{2}\right) \\
& +\int_{p_{t}^{2}}^{k_{\max }^{2}} d^{2} k_{1 t} d^{2} k_{2 t} F\left(x_{1}, k_{1 t}^{2} ; \mu^{2}\right) \\
& \times F\left(x_{2}, k_{2 t}^{2} ; \mu^{2}\right) \hat{\sigma}\left(x_{1} x_{2} s, k_{1 t}^{2}, k_{2 t}^{2}, p_{t}^{2}\right) \\
= & I^{c f}+I^{c t} .
\end{aligned}
$$

The off-shell cross section is built in order to give the usual on-shell cross section in the limit $k_{i t}^{2} \ll p_{t}^{2}$. Then the first term above can be approximately written

$$
\begin{aligned}
I^{c f}= & \hat{\sigma}\left(x_{1} x_{2} s, p_{t}^{2}\right) \int^{p_{t}^{2}} d^{2} k_{1 t} d^{2} k_{2 t} F\left(x_{1}, k_{1 t}^{2} ; \mu^{2}\right) \\
& \times F\left(x_{2}, k_{2 t}^{2} ; \mu^{2}\right) .
\end{aligned}
$$

Here $\hat{\sigma}\left(x_{1} x_{2} s, p_{t}^{2}\right)$ refers to the on-shell cross section (since it has no $k_{i t}^{2}$ dependence). Finally, using the definition (11), we obtain

$$
I^{c f}=f\left(x_{1}, p_{t}^{2} ; \mu^{2}\right) f\left(x_{2}, p_{t}^{2} ; \mu^{2}\right) \hat{\sigma}\left(x_{1} x_{2} s, p_{t}^{2}\right) .
$$

Comparing this expression with Eq. (3), we see that it corresponds to the collinear factorization formula with the choice $Q^{2}=p_{t}^{2}$.

Splitting the integral at $2 p_{t}^{2}$ instead of $p_{t}^{2}$ will not change anything for the $k_{t}$-factorization, while the collinear factorization part will be given by

$$
I^{c f}=f\left(x_{1}, 2 p_{t}^{2} ; \mu^{2}\right) f\left(x_{2}, 2 p_{t}^{2} ; \mu^{2}\right) \hat{\sigma}\left(x_{1} x_{2} s, p_{t}^{2}\right),
$$

showing that there is an uncertainty on the choice of the hard scale. This procedure, which explains why $p_{t}^{2}$ appears in the parton densities, makes sense only if the second term in Eq. (17) gives a correction. Formally, there is an uncertainty on the choice of the scale $Q^{2}$ making the second integral in Eq. (17) small:

$$
\begin{aligned}
I^{c t}= & \int_{Q^{2}}^{k_{t, \max }^{2}} d^{2} k_{1 t} d^{2} k_{2 t} F\left(x_{1}, k_{1 t}^{2} ; \mu^{2}\right) F\left(x_{2}, k_{2 t}^{2} ; \mu^{2}\right) \\
& \times \hat{\sigma}\left(x_{1} x_{2} s, k_{1 t}^{2}, k_{2 t}^{2}, p_{t}^{2}\right) \ll f\left(x_{1}, Q^{2} ; \mu^{2}\right) \\
& \times f\left(x_{2}, Q^{2} ; \mu^{2}\right) \hat{\sigma}\left(x_{1} x_{2} s, p_{t}^{2}\right) .
\end{aligned}
$$

Note that $Q^{2}$ can be interpreted as the effective upper bound for $k_{t}^{2}$ integration.

To summarize this section, we can rewrite Eq. (17) as

$$
I()=I^{c f}\left(Q^{2}\right)+I^{c t}\left(Q^{2}\right),
$$

with $I^{c f}$ the collinear factorization contribution and $I^{c t}$ a correction term. An appropriate hard scale fulfills the relation $I^{c t}\left(Q^{2}\right) \ll I^{c f}\left(Q^{2}\right)$, and its choice is not unique. This is the first main result of this paper. The uncertainty on this choice is not taken into account in current calculations and could be numerically large compared to the factorization scale uncertainty, the latter being reduced by higher-order calculations.
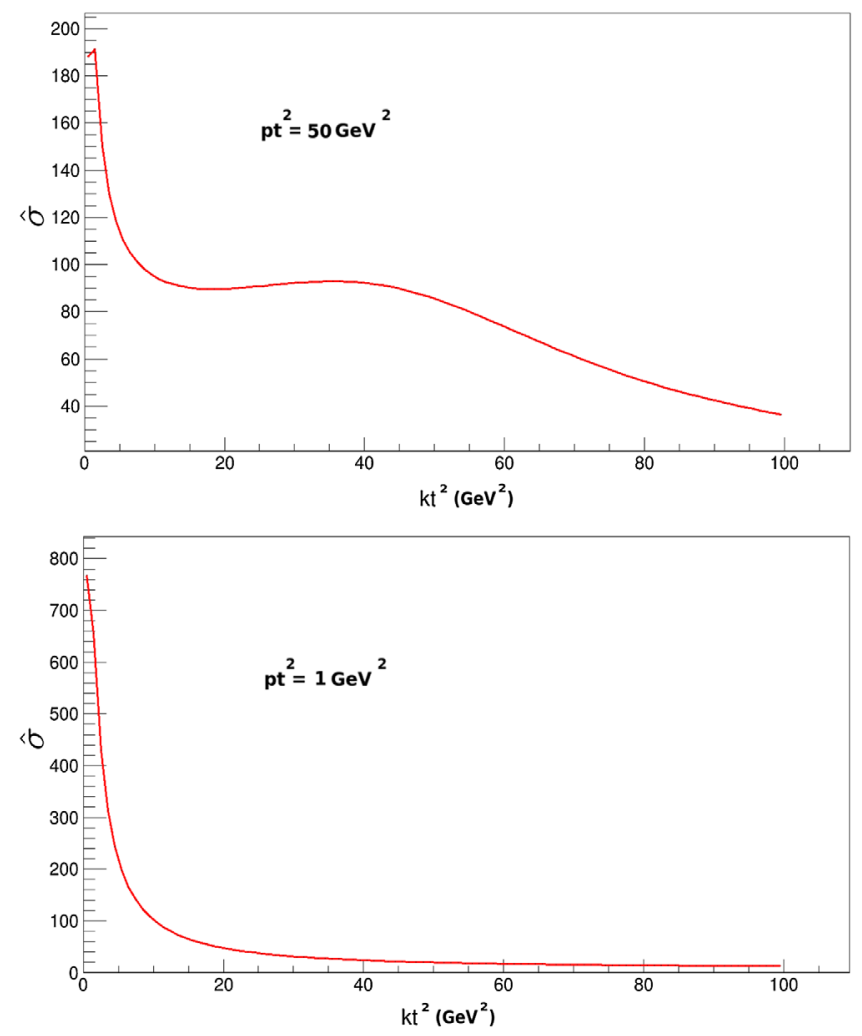

FIG. 2. Off-shell cross section for the process $g g \rightarrow Q \bar{Q}$ (taken from Ref. [2]) as a function of the transverse momentum $k_{t}^{2}=$ $k_{1 t}^{2}=k_{2 t}^{2}$ of the incoming spacelike partons. Top: For central rapidity, $y=0$, and $p_{t}^{2}=50$. Bottom: $y=0, p_{t}^{2}=1$. Other variables have been integrated out.

The 1.h.s. of Eq. (22) does not depend on $Q^{2}$. As explained in the beginning of this section, the hard scale uncertainty is absent in the $k_{t}$-factorization formalism.

\section{CHOOSING THE HARD SCALE}

We will now explain qualitatively why $Q^{2}=p_{t}^{2}$ is an acceptable choice, making the collinear factorization formula accurate [in the sense that Eq. (21) is true], even at small transverse momentum. To understand this, we will consider separately the cases of high $p_{t}$ and low $p_{t}(\sim 1 \mathrm{GeV})$.

The reason why the integral $I^{c t}$ can be small even if the phase space for integration is large is due to the fact that in the region $1 \ll p_{t}^{2}<k_{i, t}^{2}<k_{t, \text { max }}^{2}$ the off-shell cross section is slowly decreasing with $k_{t}^{2}$ (factor 2 between 0 and $40 \mathrm{GeV}^{2}$; see Fig. 2, upper panel), while the unintegrated gluon density is strongly suppressed (by a power of $k_{t}^{2}$ ). ${ }^{5}$

Consequently, in the high $p_{t}$ case, what matters is to integrate up to a large scale, which can be $Q^{2}=p_{t}^{2}$ but also $Q^{2}=4 p_{t}^{2}$. In any case, all of this kinematical region is suppressed by the unintegrated parton densities. The small

\footnotetext{
${ }^{5}$ At small $x$. For $x>x_{0} \sim 0.01$, the suppression is even exponential.
} 
sensitivity of the result to this scale was expected from the $\ln \left(Q^{2} / \mu^{2}\right)$ behavior of parton densities; see Eq. (4).

At small $p_{t}$, the suppression due to the unintegrated gluon density is not enough to explain why Eq. (21) is true if one chooses $Q^{2}=p_{t}^{2}$. But, in this region, the off-shell cross section decreases quickly with $k_{t}^{2}$ (Fig. 2, lower panel), making the integration up to $\sim p_{t}^{2}$ sufficient.

This is our second main result. The choice $Q^{2}=p_{t}^{2}$ for the hard scale is explained by the dynamical behavior of the unintegrated parton densities and the off-shell cross section. The role of the off-shell cross section in choosing the effective cutoff for the $Q^{2}$ integration in DIS has been underlined in Ref. [10].

\section{CONCLUSION}

We have seen that, by choosing correctly $Q^{2}$ in Eq. (21), the $k_{t}$-factorization formula can be split into two parts: the collinear factorization plus a correction term. In this case, the scale $Q^{2}$ can be interpreted as the effective upper bound for the $k_{t}^{2}$ integration. Based on the behavior of the unintegrated gluon density and the off-shell cross section, we argued that in the case of hadron-hadron collisions the choice $Q^{2}=p_{t}^{2}$ can be made, but it is not unique.

Consequently, there is an uncertainty coming from the choice of the hard scale. The difference with the uncertainty on the factorization scale is that it is not reduced by higherorder corrections, the reason being that it does not obey a renormalization group equation. This uncertainty is absent in the $k_{t}$-factorization, thanks to the integration on the transverse momentum.

In arriving at this conclusion, we used quite general arguments, and our results can be easily extended to other cases where the factorization formulas are valid.

A practical consequence is that the uncertainty estimation within the collinear factorization is underestimated (usually, the estimation of uncertainties is done for the mass, the parton densities, and the factorization scale). Note that, instead of $Q^{2}=p_{t}^{2}$, we could choose this scale in order to keep the correction term at $1 \%$ (for instance). Then we can expect a more complicated relation $Q^{2}=f\left(p_{t}^{2}\right)$. In particular, one should have $Q^{2}>p_{t}^{2}$ at small $p_{t}$, since this is the kinematical region where the collinear factorization is less accurate.

\section{ACKNOWLEDGMENTS}

We thank F. Hautmann, H. Jung, and J. Bartels for useful discussions and valuable comments. We are also grateful to E. Levin for interesting discussions. We acknowledge support from Chilean FONDECYT Grant No. 3160493. We acknowledge support by the Basal Project No. FB0821.
[1] J. C. Collins and R. K. Ellis, Heavy-quark production in very high energy hadron collisions, Phys. Rev. B 360, 3 (1991).

[2] S. Catani, M. Ciafaloni, and F. Hautmann, High energy factorization and small- $x$ heavy flavour production, Phys. Rev. B 366, 135 (1991).

[3] L. Gribov, E. Levin, and M. Ryskin, Semihard processes in QCD, Phys. Rep. 100, 1 (1983).

[4] E. M. Levin, M. G. Ryskin, Y. M. Shabelski, and A. G. Shuvaev, Heavy quark production in semihard nucleon interactions, Sov. J. Nucl. Phys. 53, 657 (1991).

[5] E. Kuraev, L. Lipatov, and V. Fadin, Multiregge processes in the Yang-Mills theory, Sov. Phys. JETP 44, 443 (1976); The Pomeranchuk singularity in nonabelian gauge theories, Sov. Phys. JETP 45, 199 (1977); I. Balitsky and L. Lipatov, The pomeranchuk singularity in quantum chromodynamics, Sov. J. Nucl. Phys. 28, 822 (1978).

[6] R. K. Ellis, W. J. Stirling, and B. R. Webber, $Q C D$ and Collider Physics (Cambridge University Press, Cambridge, England, 1996).

[7] B Anderson et al., Small $x$ Phenomenology: Summary and Status, arXiv:hep-ph/0204115v2.

[8] J. Collins, D. Soper, and G. Sterman, Factorization of Hard Processes in QCD, arXiv:hep-ph/0409313.

[9] V. N. Gribov and L. N. Lipatov, Deep inelastic e p scattering in perturbation theory, Sov. J. Nucl. Phys. 15,
438 (1972); G. Altarelli and G. Parisi, Asymptotic freedom in parton language, Nucl. Phys. B126, 298 (1977); Y. L. Dokshitzer, Calculation of the structure functions for deep inelastic scattering and $e^{+} e^{-}$annihilation by perturbation theory in quantum chromodynamics, Sov. Phys. JETP 46, 641 (1977).

[10] S. Catani, M. Ciafaloni, and F. Hautmann, Leptoproduction of heavy flavour at high energies, Nucl. Phys. B, Proc. Suppl. 29A, 182 (1992).

[11] S. Catani, M. Ciafaloni, and F. Hautmann, in Proceedings of Physics at HERA, Hamburg, 1991 (CERN, Geneva, 1992), Vol. 2, TH. 6398, pp. 690-711.

[12] A. V. Lipatov, V. A. Saleev, and N. P. Zotov, Heavy Quark Production at the TEVATRON in the Semihard QCD Approach and the Unintegrated Gluon Distribution, arXiv:hep$\mathrm{ph} / 0112114$.

[13] Yu. M. Shabelski, A. G. Shuvaev, and I. V. Surnin, Heavy quark production in $k_{t}$ factorization approach at LHC energies, Int. J. Mod. Phys. A 33, 1850003 (2017).

[14] M. A. Kimber, A. D. Martin, and M. G. Ryskin, Unintegrated parton distributions, Phys. Rev. D 63, 114027 (2001).

[15] S. Catani, M. Ciafaloni, and F. Hautmann, High-energy factorization in QCD and minimal subtraction scheme, Phys. Lett. B 307, 147 (1993). 